This item was submitted to Loughborough's Research Repository by the author.

Items in Figshare are protected by copyright, with all rights reserved, unless otherwise indicated.

\title{
Quasi-superradiant soliton state of matter in quantum metamaterials
}

PLEASE CITE THE PUBLISHED VERSION

https://doi.org/10.1140/epjb/e2017-80567-7

PUBLISHER

(c) EDP Sciences, Societa Italiana di Fisica, Springer-Verlag

VERSION

AM (Accepted Manuscript)

PUBLISHER STATEMENT

This work is made available according to the conditions of the Creative Commons Attribution-NonCommercialNoDerivatives 4.0 International (CC BY-NC-ND 4.0) licence. Full details of this licence are available at: https://creativecommons.org/licenses/by-nc-nd/4.0/

\section{LICENCE}

CC BY-NC-ND 4.0

\section{REPOSITORY RECORD}

Asai, Hidehiro, Shiro Kawabata, Sergey Saveliev, and Alexandre M. Zagoskin. 2019. "Quasi-superradiant Soliton State of Matter in Quantum Metamaterials”. figshare. https://hdl.handle.net/2134/27967. 


\title{
Quasi-superradiant soliton state of matter in quantum metamaterials ${ }^{\star}$
}

\author{
Hidehiro Asai ${ }^{1,2, a}$, Shiro Kawabata ${ }^{1}$, Sergey E. Savel'ev ${ }^{2}$, and Alexandre M. Zagoskin ${ }^{2,3}$ \\ ${ }^{1}$ Nanoelectronics Research Institute (NeRI), National Institute of Advanced Industrial Science and Technology \\ (AIST), Tsukuba, Ibaraki 305-8568, Japan \\ 2 Department of Physics, Loughborough University, Leicestershire LE11 3TU, UK \\ 3 Theoretical Physics and Quantum Technologies Department, Moscow Institute for Steel and Alloys, 119049 \\ Moscow, Russia
}

Received 8 October 2017

Published online (Inserted Later) - C EDP Sciences, Società Italiana di Fisica, Springer-Verlag 2017

\begin{abstract}
Strong interaction of a system of quantum emitters (e.g., two-level atoms) with electromagnetic field induces specific correlations in the system accompanied by a drastic increase of emitted radiation (superradiation or superfluorescence). Despite the fact that since its prediction this phenomenon was subject to a vigorous experimental and theoretical research, there remain open question, in particular, concerning the possibility of a first order phase transition to the superradiant state from the vacuum state. In systems of natural and charge-based artificial atom this transition is prohibited by "no-go" theorems. Here we demonstrate numerically and confirm analytically a similar transition in a one-dimensional quantum metamaterial - a chain of artificial atoms (qubits) strongly interacting with classical electromagnetic fields in a transmission line. The system switches from vacuum state to the quasi-superradiant (QS) phase with one or several magnetic solitons and finite average occupation of qubit excited states along the transmission line. A quantum metamaterial in the QS phase circumvents the "no-go" restrictions by considerably decreasing its total energy relative to the vacuum state by exciting nonlinear electromagnetic solitons.
\end{abstract}

\section{Introduction}

2 The interaction of light and matter in artificial optical media is the focus of a significant research effort (see e.g., [1-6]). The strong light-matter interaction in such systems make possible such effects as unusual photon collapse-and-revivals [7], Schrödinger-cat states [8], non-classical radiation [9], unusual Casimir effect and pseudo-vacuum states [10]. For a subclass of these media with extended spatial quantum coherence (quantum metamaterials [11-19]) a number of novel phenomena are predicted, which do not have place in natural materials and classical metamaterials. This adds a new dimension to the long-standing discussion about the possibility of a superradiant transition in the system of atoms strongly interacting with electromagnetic waves in a cavity [20-26]. In particular, reference [25] extends the "no-go" theorem to circuit QED with charge (but not flux) qubits.

It was predicted $[20,21]$ that in a cavity containing many two-level atoms there exists a possibility of a transition from the vacuum state (with no photons and all atoms being in the ground state) to the superradiant state (with nonzero photon occupation number accompanied by

\footnotetext{
* Supplementary material in the form of one pdf file from the Journal web page at

https://doi.org/10.1140/epjb/e2017-80567-7.

a e-mail: hd-asai@aist.go.jp
}

atom excitations) as the light-atom interaction increases. This topic remained subject of vigorous investigation ever since $[27,28]$. Soon after this prediction it was pointed out that the predicted transition is "an interesting artefact" [22], caused by neglecting the term quadratic in the electromagnetic vector potential in the light-matter interaction Hamiltonian. Taking it into account eliminates the superradiant state. This "no-go" theorem (see e.g., [22-24]) was claimed not to apply to both bosonic and fermionic artificial-atom arrays (in particular, in case of circuit QED), at least when driven by a laser to a non-equilibrium state $[26,29-32]$. Nevertheless in [25] the theorem was restated for charge-qubit based circuit-QED systems. The precise requirements to a system capable of undergoing the superradiant phase transition $[25,26]$ and possible connections between the superradiance and similar phenomena such as the dynamical Casimir effect $[33,34]$ or the essentially non-classical spontaneous radiation $[9,10]$ are still being vigorously debated, and the investigation of these and related phenomena in artificial structures is highly relevant.

A quantum metamaterial (QMM) is a globally quantum coherent array of artificial atoms with a limited control of their quantum state [12]. In this article we consider superradiant-like transition in the presence of external magnetic field in such a structure. Though the basic properties of a QMM are qualitatively independent on its specific realization $[18,19]$, here we use a model of a 

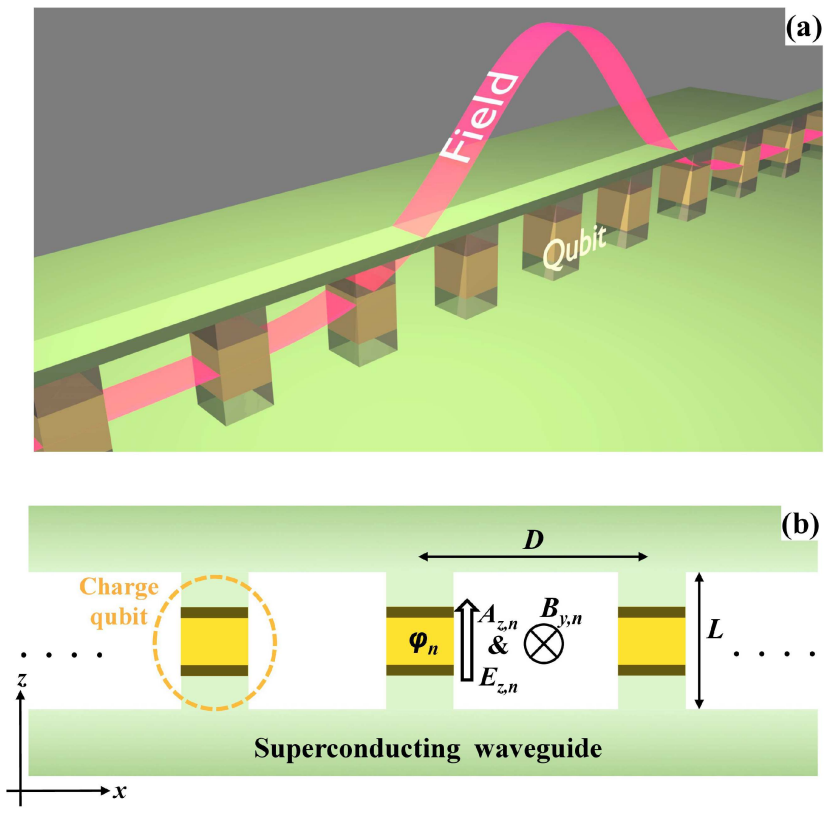

Fig. 1. One-dimensional quantum metamaterial: (a) a chain of superconducting charge qubits playing the role of artificial atoms in a Josephson transmission line formed between two superconducting banks connected via superconducting islands (charge qubits). The (green) wave represent distribution of the electromagnetic field in the structure. (b) The schematic view of the structure. The magnetic field penetrates through the openings between the superconducting islands. The electric and magnetic fields and the vector potential are assumed constant within each opening. also generates structural transitions between states with different number of solitons. When cycling the external magnetic field around zero, the soliton number can be either zero or nonzero at $H_{\text {ext }}=0$ resulting in a rich variety of remagnetization loops associated with quantum pinning of solitons on the spatial variations of the qubit level occupation which, in turn, is generated by the solitons themselves.

\section{Model}

The system in question is a quantum counterpart of the standard Josephson transmission line [35] where Josephson junctions linking two superconducting banks are replaced by charge qubits (Fig. 1), i.e., small superconducting islands connected to both long superconducting banks with high-resistance tunneling junctions $(R>$ $R_{Q} \approx 12 \mathrm{k} \Omega$ ), and with controlled potential bias with respect to the banks [36]. The quantum states of an island differ by the number of extra Cooper pairs on them, and are coupled to the electromagnetic field through their electric charge.

As in [11] we describe qubits quantum-mechanically while treating the electromagnetic fields in the transmission line classically (in line with the standard semiclassical approach to atom-field interactions, valid in case of strong enough fields [37]). We direct the vector potential $A_{z}$ across the junctions (along $z$-axis) and assume it to be constant in each "cell" between two adjacent qubits.

We start from the classical expression for the system's total (electromagnetic, electrostatic and Josephson) energy as a function of the phases $\phi_{n}$ of the superconducting order parameters on the islands: $\mathcal{E}_{\text {total }}=\sum_{n} \mathcal{E}_{n}$ with

$$
\begin{aligned}
\mathcal{E}_{n}= & \frac{C}{2} \frac{\hbar^{2}}{4 e^{2}}\left[\left(\frac{d \phi_{n}}{d t}+\frac{\pi D}{\Phi_{0}} \frac{d A_{z, n}}{d t}\right)^{2}\right. \\
& \left.+\left(\frac{d \phi_{n}}{d t}-\frac{\pi D}{\Phi_{0}} \frac{d A_{z, n}}{d t}\right)^{2}\right] \\
& -E_{J}\left[\cos \left(\phi_{n}+\frac{\pi D A_{z, n}}{\Phi_{0}}\right)+\cos \left(\phi_{n}-\frac{\pi D A_{z, n}}{\Phi_{0}}\right)\right] \\
& +\frac{8 \pi}{D L}\left[\frac{A_{z, n+1}-A_{z, n}}{L}\right]^{2}
\end{aligned}
$$

Here, the index $n$ corresponds to the qubit number in the chain, $C$ is the junction capacitance, $\Phi_{0}=h c / 2 e$ is the flux quantum, $E_{J}=\frac{I_{c} \Phi_{0}}{2 \pi c}$ is the Josephson coupling energy, $I_{c}$ is the critical current of the Josephson junctions linking the qubits to the superconducting busbars, $D$ and $L$ are distances between the neighbouring qubits and between the two superconducting banks, respectively. It is convenient to use dimensionless variables: the inter-qubit distance $l=L / \lambda$, vector potential $a_{z, n}=\pi D A_{z, n} / \Phi_{0}$, time $\tau=\omega_{J} t$ and energy $E_{n}=\mathcal{E}_{n} / E_{J}$, where the Josephson plasma frequency $\omega_{J}=2 e I_{c} /(\hbar C)$ and the effective spatial scale $\lambda=c / \omega_{J}$. Then the energy per unit is

$$
\begin{aligned}
E_{n}= & \left(\frac{d \phi_{n}}{d \tau}\right)^{2}+\left(\frac{d a_{z, n}}{d \tau}\right)^{2}-2 \cos \phi_{n} \cos a_{z, n} \\
& +\beta^{2}\left(\frac{a_{z, n+1}-a_{z, n}}{l}\right)^{2} .
\end{aligned}
$$


The quantization is performed by replacing the phases $\phi_{n}$ and their conjugate canonical momenta with operators [38] and yields the Schrödinger equation

$$
i \hbar \frac{d}{d t}\left|\Psi_{n}^{s}\right\rangle=\mathcal{H}_{n}^{q u b i t}\left|\Psi_{n}^{s}\right\rangle=\left(\mathcal{H}_{n, i n t}^{q u b i t}+\mathcal{H}_{n, 0}^{q u b i t}\right)\left|\Psi_{n}^{s}\right\rangle,
$$

with the qubit Hamiltonian $\mathcal{H}_{n, 0}^{\text {qubit }}$ and field-qubit interaction $\mathcal{H}_{n, \text { int }}^{\text {qubit }}$ :

$$
\mathcal{H}_{n, 0}^{q u b i t}=-\frac{e^{2}}{C} \frac{\partial^{2}}{\partial \phi_{n}^{2}}-2 E_{J} \cos \phi_{n}
$$

1

$$
\mathcal{H}_{n, \text { int }}^{q u b i t}=2 E_{J} \cos \phi_{n}\left(1-\cos a_{z, n}\right) .
$$

We restrict our analysis to the two first energy levels of each island (which is justified for a realistic choice of charge qubit parameters). We will also neglect entanglement between qubits and seek the qubits' wave function in the factorized form: $\left|\Psi_{n}^{s}\right\rangle=C_{0}^{n}(t) e^{-\frac{i \mathcal{E}_{0} t}{\hbar}}|0\rangle+$ $C_{1}^{n}(t) e^{-\frac{i \varepsilon_{1} t}{\hbar}}|1\rangle .{ }^{1}$ Then the Schrödinger equation for qubits reduces to

$$
\begin{aligned}
i \frac{d}{d \tau} C_{0}^{n}(\tau) & =s\left(1-\cos a_{z, n}\right) C_{1}^{n}(\tau) e^{-i s \epsilon \tau}, \\
i \frac{d}{d \tau} C_{1}^{n}(\tau) & =s\left(1-\cos a_{z, n}\right) C_{0}^{n}(\tau) e^{i s \epsilon \tau},
\end{aligned}
$$

where $s=E_{J} / \hbar \omega_{J}, \epsilon$ is the dimensionless excitation energy (energy difference between the first and the ground levels [38]).

Equations for the electromagnetic field in the transmission line can be derived by taking the expectation value of the total energy (1) in the quantum state of the qubit subsystem. By using Hamilton's equations for the dimensionless vector potential, we derive the effective sine-Gordon equation for the line $[11,16]$ :

$$
\begin{aligned}
\beta^{2} \frac{a_{z, n+1}+a_{z, n-1}-2 a_{z, n}}{l^{2}}= & \frac{d^{2} a_{z, n}}{d \tau^{2}}+V_{n} \sin a_{z, n} \\
& +\gamma \frac{d a_{z, n}}{d \tau}
\end{aligned}
$$

$$
V_{n}(\tau)=\frac{1}{2}\left(C_{0}^{n *}(\tau) C_{1}^{n}(\tau) e^{-i s \epsilon \tau}+C_{0}^{n}(\tau) C_{1}^{n *}(\tau) e^{i s \epsilon \tau}\right)
$$

where we have added the phenomenological damping term $\gamma d a_{z, n} / d \tau$ (which can be introduced in the quantum Routh formalism [38]). This equation is quite similar to the standard discrete sine-Gordon equation (see e.g., in Ref. [39]) for a classical Josephson transmission line, but

\footnotetext{
${ }^{1}$ Individual qubits in our QMM can on be entangled through their interaction mediated by the electromagnetic field in the system. This entanglement will be negligible if the number of photons in the field is large (see arXiv:cond-mat/0207214), and is irrelevant to the quantum phenomena which requires only coherence such as superradiance.
}

with the key difference that the "effective critical Josephson current", $V_{n}$, now depends on the quantum states of the charge qubits.

\section{Results}

First we consider which state the system settles in at zero magnetic field $H_{\text {ext }}=0$, imposing boundary conditions $H_{\text {ext }}=\left(a_{z, 1}-a_{z, 0}\right) / l=\left(a_{z, N}-a_{z, N-1}\right) / l=0$. Here $N$ is the total number of qubits in the system. Surprisingly, the vacuum state with $C_{1}^{n}=0, C_{0}^{n}=1, a_{z, n}=0$ is stable only for relatively weak qubit-field couplings. Then the vacuum state becomes unstable, and the system evolves to the quasi-superradiant state with one or several solitons spontaneously arising (see Supplemental Materials for the comparison with an analytical calculation. ${ }^{2}$ Even though the energy of the qubit system itself and the energy of the magnetic field both increase (Fig. 2c), the total energy of this quasi-superradiant (QS) state decreases due to interactions between the field and the qubits. Figure 2a shows the magnetic field and vector potential distribution in the QS state. Since the vector potential changes from $-\pi$ to $\pi$ along the quantum transmission line, we conclude that a magnetic field soliton carries one flux quantum similarly to a usual Josephson vortex in a standard long Josephson junction (see e.g., [40]). However, the QS state has a more complex structure than a Josephson vortex, since the soliton-like fields' distribution is here accompanied with a spatial modulation of qubit state occupation probabilities $C_{0}^{n}$ and $C_{1}^{n}$ (Fig. 2b). Here the macroscopic, classical magnetic soliton depends on the quantum state of the qubits. In contrast to the standard superradiant state of two-level atoms in a cavity interacting with one or few modes, the soliton is an essentially nonlinear magnetic field distribution arising via a complex interaction of a very large number of field-modes.

In zero magnetic field the transition to the QS state breaks the system's symmetry by spontaneously choosing the soliton polarity (positive or negative). The external magnetic field eliminates the spontaneous degeneracy of the QS phase, and the transition point from the Meissner state with no solitons to the soliton state is shifted: the critical coupling for the transition becomes a function of $\left.H_{e x t}\right)$. Figure 3 shows the evolution of the metamaterial phases when varying the external magnetic field. We consider the case when the vacuum state $\left(a_{z, n}=0\right)$ is stable at $H_{\text {ext }}=0$, but it becomes unstable for quite a weak $H_{\text {ext }}$ resulting in the formation of a one-soliton state (Fig. 3, main panel - the remagnetization loop and magnetic field distribution (A)). With a further increase of the external magnetic field $H_{\text {ext }}$ a sequence of the structural transitions to the phases with larger numbers of solitons occur accompanied with jumps in the trapped magnetic flux $\Phi$. When the external field $H_{\text {ext }}$ decreases from a certain maximal value, other sequence of the structural transitions occurs first decreasing the number of the "positive-polarity" solitons followed by formation and increasing the number of

\footnotetext{
${ }^{2}$ See also the supplemental material for the analytical solution of

the soliton state in strong coupling limit.
} 


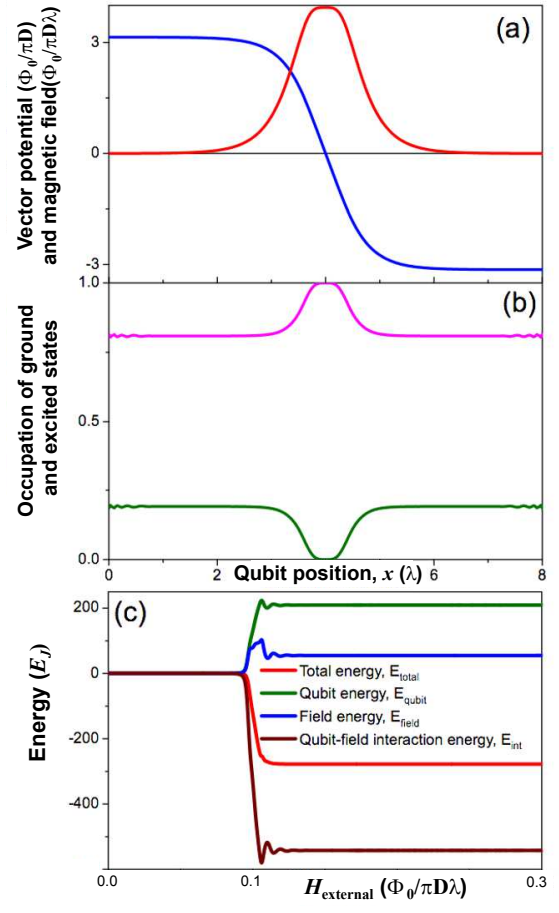

Fig. 2. Quasi-superradiant phase: (a) distribution of dimensionless vector potential $a_{z, n}$ and magnetic field in the quantum Josephson transmission line. (b) Distribution of occupation probabilities for ground (bottom (green) curve) and excited (upper (magenta) curve) qubit states, the strong variation of the qubit state occupation occurs at the soliton center resulting in pinning of the soliton. (c) Evolution of the qubit energy $E_{\text {qubit }}$, the field energy $E_{\text {field }}$, and the qubit-field interaction energy $E_{\text {int }}$ as a function of the weak external magnetic field $H_{\text {ext }}$. Even though the field and qubit energies, $E_{\text {qubit }}$ and $E_{\text {field }}$, both increase at the transition, the total energy of the quasi-superradiant phase, $E_{\text {total }}$, decreases due to a sharp drop of the interaction energy, $E_{\mathrm{int}}$. Parameters used in simulations are: $s=1, \beta=0.25, \epsilon=\pi, l=L / \lambda=0.05, \gamma=0.25$ and the total number of qubits is 400 .

the "anti-solitons" or solitons with the opposite magnetic field direction.

It is worth noting that, at zero external field $H_{\text {ext }}=0$, the system still keeps one soliton on the steady remagnetization curve (external (blue) loop in Fig. 3). This indicates that the formally stable initial Meissner state with zero solitons in the system is actually a metastable state. Thus, the vacuum state in this case is metastable in contrast to the QS ground state (which can be called the "dressed-vacuum" state) for this value of the fieldqubit coupling. This transition is analogue of the first order transition associated with the vortex penetration into type II superconductors at lower critical field. The role of qubit-field coupling strength can be here compared to that of the critical field, at which the vortex penetration in the system becomes energetically profitable. Therefore, the transition from the vacuum to the QS phase in onedimensional quantum metanaterials is the first rather than the second order phase transition [21].

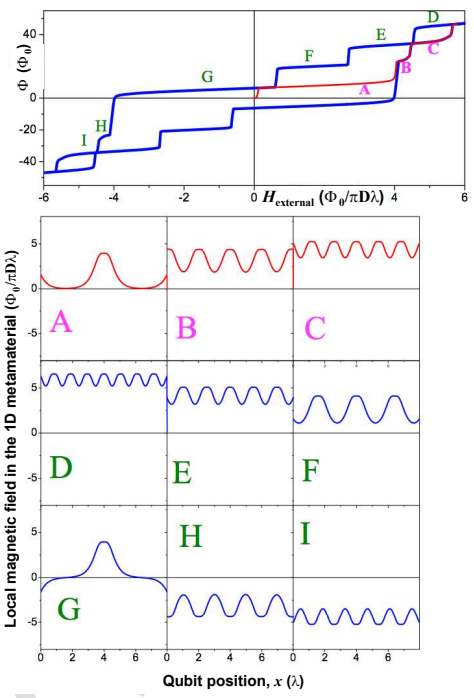

Fig. 3. Remagnetization loop as a sequence of the structural transitions: (a) evolution of the total flux $\Phi$ trapped in the quantum transmission line as a function of the external magnetic field $H_{\text {ext }}$. The (red) curve ABC starting at origin is the "virgin curve" which the system passes only once after its initialization in the vacuum state at zero external field, $H_{\text {ext }}=0$. Applying a very weak external field results in the transition to the one-soliton phase (panel A) which then goes through a sequence of transitions to the phases with 3 (panel B), 5 (panel C) and 7 (panel D) solitons. On the returning branch the system passes through the states with 5 (panel E), 3 (panel F), 1 (panel G), -3 (i.e., three anti-soliton) (panel H), -5 (five antisolitons) (panel I) and -7 solitons. repeated cycling of $H_{\text {ext }}$ forms the steady state (blue) loop CD ... I ... B. Note that the state with one soliton or one anti-soliton is the ground state at $H_{\text {ext }}=0$ with spontaneous symmetry breaking to either 1 or -1 soliton. All parameters are the same as in Figure 2, while the sweeping rate $d H_{\text {ext }} / d t=2.5 \times 10^{-4} \omega_{J} \Phi_{0} / \pi D \lambda$.

Finally we consider the dependence of the QS transition on the sample size (the number of qubits in the quantum Josephson transmission line). Using the same set of parameters as in Figure 3, we simulate the steady remagnetization curve $\Phi\left(H_{\text {ext }}\right)$ for a shorter chain (see Fig. 4). As one can see, the state at zero external field is always the vacuum (or the Meissner phase) with no solitons in the sample. Only at a high enough external magnetic field $H_{\text {ext }}$, the soliton state (here with a single soliton) can be formed, but it becomes unstable with decreasing $H_{\text {ext }}$ (on the returning branch of the remagnetization curve) before $H_{\text {ext }}$ drops to zero.

Remarkably, the unusual "butterfly-like" loops similar to those obtained here appear in several apparently different systems, including crossing vortex lattices [41], magnetic vortices in nano-discs [42] and thermal atomic switches [43]. For all such systems the "butterfly-like" loops originate either due to a nontrivial interplay of fluctuations with driving or due to the complex nature of vortex pinning in the bulk and on surface. In our particular case, the QMMs provide a "quantum pinning" for Josephson-like vortices on the inhomogeneity formed 


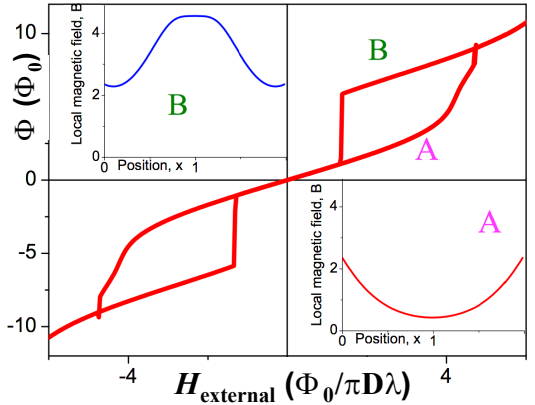

Fig. 4. "Butterfly" remagnetization curve: $\Phi\left(H_{\text {ext }}\right)$ dependence for the same parameters as in Figure 3, but for a shorter transmission line (100 qubits). Note that there is no soliton phase at zero external magnetic field, $\Phi\left(H_{\text {ext }}=0\right)=0$ (see inset A for the Meissner phase). Transition to the one-soliton phase (inset B) occurs at a relatively high magnetic field. On the return branch, the soliton phase switches back to the Meissner phase before the external field $H_{\text {ext }}$ drops to zero.

by fluctuating qubit occupation numbers of ground and excited states.

\section{Conclusions}

We predict a new state of matter for QMMs - the quasisuperradiant soliton phase - when the coupling between electromagnetic fields and qubits crosses a threshold, which can be tuned by the external fields resulting in a series of structural superrradiance transitions and a variety of remagnetization loops. It is worthy of note that recent studies have also shown the appearance of the superradiant phase transition in superconducting circuit QED systems composed of a huge number of Josephson junctions both theoretically [44] and experimentally [45]. The seeming violation of the "no-go" theorem for charge-based circuit QED can be attributed to the more complex structure of qubit coupling to the field modes in the system.

The analogy of the predicted effects to vortex penetration in a wide Josephson junction can be taken further: the key component of both phenomena is phase coherence along the junction, which in case of the Josephson effect is provided by the superconducting coupling (i.e., dynamically), while in our case it is maintained simply due to the long enough decoherence time of individual qubits. It can be therefore extended to non-superconducting quantum metamaterials as well as to other meso- and macroscopic artificial quantum coherent systems.

SES acknowledge support from Leverhulme Trust. AMZ was supported in part by the EPSRC grant EP/M006581/1 and by the Ministry of Education and Science of the Russian Federation in the framework of Increase Competitiveness Program of NUST MISiS (No. K2-2014-015). H.A. was partially supported by a Grant-in-Aid for Young Scientists (B) from JSPS (Grant No. 26790062). S.K was partially supported by a Grant-in-Aid for Scientific Research (C) from JSPS (Grant No. 24510146).

\section{Author contribution statement}

H.A., A.M.Z., and S.S. formulated the mathematical model of the quantum metamaterial. H.A. and S.K performed numerical and analytical calculations for the theoretical models. All authors contributed to the discussion of the physical content of the investigated phenomena and their possible applications, and to the writing of the paper.

\section{References}

1. A. Wallraff et al., Nature 431, 162 (2004)

2. G. Günter et al., Nature 479, 376 (2011)

3. T. Niemczyk et al., Nat. Phys. 6, 772 (2010)

4. Y. Todorov, A.M. Andrews, R. Colombelli, S. DeLiberato, C. Ciuti, P. Klang, G. Strasser, C. Sirtori, Phys. Rev. Lett. 105, $196402(2010)$

5. T. Schwartz, J.A. Hutchison, C. Genet, T.W. Ebbesen, Phys. Rev. Lett. 106, 196405 (2011)

6. A.J. Hoffman, S.J. Srinivasan, S. Schmidt, L. Spietz, J. Aumentado, H.E. Türeci, A.A. Houck, Phys. Rev. Lett. 107, 053602 (2011)

7. J. Casanova, G. Romero, I. Lizuain, J.J. García-Ripoll, E. Solano, Phys. Rev. Lett. 105, 263603 (2010)

8. S. Ashhab, F. Nori, Phys. Rev. A 81, 042311 (2010)

9. A. Ridolfo, S. Savasta, M.J. Hartmann, Phys. Rev. Lett. 110, 163601 (2013)

10. R. Stassi, A. Ridolfo, O. Di Stefano, M.J. Hartmann, S. Savasta, Phys. Rev. Lett. 110, 243601 (2013)

11. A.L. Rakhmanov, A.M. Zagoskin, S. Savel'ev, F. Nori, Phys. Rev. B 77, 144507 (2008)

12. A.M. Zagoskin, D. Felbacq, E. Rousseau, EPJ Quantum Technol. 3, 1 (2016)

13. O. Astafiev, A.M. Zagoskin, A.A. Abdumalikov, Yu.A. Pashkin, T. Yamamoto, K. Inomata, Y. Nakamura, J.S. Tsai, Science 327, 840 (2010)

14. P. Macha, G. Oelsner, J.-M. Reiner, M. Marthaler, S. André, G. Schön, U. Hübner, H.-G. Meyer, E. Il'ichev, A.V. Ustinov, Nat. Commun. 5, 5146 (2014)

15. D.S. Shapiro, P. Macha, A.N. Rubtsov, A.V. Ustinov, Photonics 2, 449 (2015)

16. H. Asai, S. Savel'ev, S. Kawabata, A.M. Zagoskin, Phys. Rev. B 91, 134513 (2015)

17. Z. Ivic, N. Lazarides, G.P. Tsironis, arXiv:1509.07662 (2015)

18. J.Q. Quach, C.H. Su, A.M. Martin, A.D. Greentree, L.C.L. Hollenberg, Opt. Express 19, 11018 (2011)

19. P.K. Jha, M. Mrejen, J. Kim, C. Wu, Y. Wang, Y.V. Rostovtsev, X. Zhang, Phys. Rev. Lett. 116, 165502 (2016)

20. R.H. Dicke, Phys. Rev. 93, 99 (1954)

21. K. Hepp, E.H. Lieb, Ann. Phys. (New York) 76, 360 (1973)

22. K. Rzaźewski, K. Wódkiewicz, W. Źacowicz, Phys. Rev. Lett. 35, 432 (1975)

23. K. Rzaźewski, K. Wódkiewicz, Phys. Rev. Lett. 96, 089301 (2006)

24. J.M. Knight, Y. Aharonov, G.T.C. Hsieh, Phys. Rev. A 17, 1454 (1978)

25. O. Viehmann, J. von Delft, F. Marquardt, Phys. Rev. Lett. 107, 113602 (2011) 
26. P. Nataf, C. Ciuti, Nat. Commun. 1, 72 (2010)

27. M. Gross, S. Haroche, Phys. Rep. 93, 301 (1982)

28. T. Brandes, Phys. Rep. 408, 315 (2005)

29. F. Dimer, B. Estienne, A.S. Parkins, H.J. Carmichael, Phys. Rev. A 75, 013804 (2007)

30. K. Baumann, C. Guerlin, F. Brennecke, T. Esslinger, Nature (London) 464, 1301 (2010)

31. J. Keeling, M.J. Bhaseen, B.D. Simons, Phys. Rev. Lett. 105, 043001 (2010)

32. J. Keeling, M.J. Bhaseen, B.D. Simons, Phys. Rev. Lett. 112, 143002 (2014)

33. G. Vacanti, S. Pugnetti, N. Didier, M. Paternostro, G.M. Palma, R. Fazio, V. Vedral, Phys. Rev. Lett. 108, 093603 (2012)

34. G.R. Berdiyorov, S. Savel'ev, M.V. Milošević, F. Kusmartsev, F.M. Peeters, unpublished

35. N.F. Pedersen, A.V. Ustinov, Supercond. Sci. Technol. 8, 389 (1995)
36. P. Caputo, M. Darula, A.V. Ustinov, H. Kohlstedt, J. 352 Appl. Phys. 81, 309 (1997)

37. D.I. Blokhintsev, Quantum mechanics (Springer, 1964)

38. A.M. Zagoskin, Quantum engineering (Cambridge University Press, 2011)

39. Z. Zheng, B. Hu, G. Hu, Phys. Rev. B 58, 5453 (1998)

40. M. Tinkham, Introduction to superconductivity (Dover Publication, Inc., New York, 1996)

41. D. Cole et al., Nat. Mater. 5, 305 (2006)

42. V. Novosad, K.Y. Guslienko, H. Shima, Y. Otani, S.G. Kim, K. Fukamichi, N. Kikuchi, O. Kitakami, Y. Shimada, Phys. Rev. B 65, 060402(R) (2002)

43. S.E. Savel'ev, F. Marchesoni, A.M. Bratkovsky, Eur. Phys. J. B 86, 501 (2013)

44. M. Bamba, K. Inomata, Y. Nakamura, Phys. Rev. Lett. 117, 173601 (2016)

45. F. Yoshihara, T. Fuse, S. Ashhab, K. Kakuyanagi, S. Saito, K. Semba, Nat. Phys. 13, 44 (2017) 


\section{Author Query}

Q1 Part labels are mentioned in the caption of Figure 3 but not provided in the art work. Please check and amend the changes.

Q2 Please provide an update for reference [34].

Q3 Please provide the publisher location for the reference $[37,38]$. 